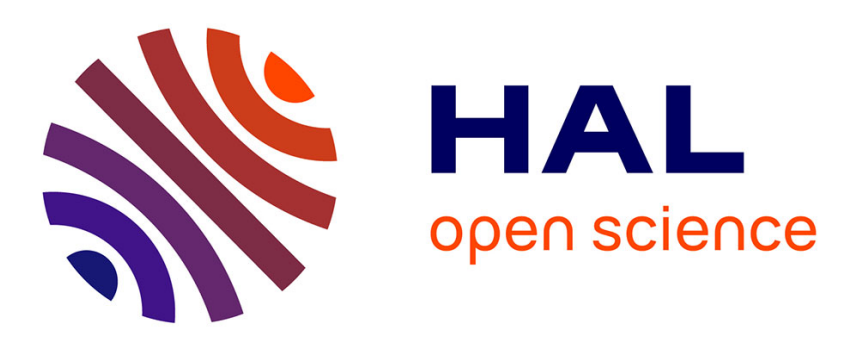

\title{
Phenomenological marine snow model for optical underwater image simulation: Applications to color restoration \\ Matthieu Boffety, Frédéric Galland
}

\section{- To cite this version:}

Matthieu Boffety, Frédéric Galland. Phenomenological marine snow model for optical underwater image simulation: Applications to color restoration. OCEANS 2012 - Yeosu, May 2012, Yeosu, South Korea. pp.1-6, 10.1109/OCEANS-Yeosu.2012.6263448 . hal-01237070

\section{HAL Id: hal-01237070 \\ https://hal.science/hal-01237070}

Submitted on 2 Dec 2015

HAL is a multi-disciplinary open access archive for the deposit and dissemination of scientific research documents, whether they are published or not. The documents may come from teaching and research institutions in France or abroad, or from public or private research centers.
L'archive ouverte pluridisciplinaire HAL, est destinée au dépôt et à la diffusion de documents scientifiques de niveau recherche, publiés ou non, émanant des établissements d'enseignement et de recherche français ou étrangers, des laboratoires publics ou privés. 


\title{
Phenomenological marine snow model for optical underwater image simulation: Applications to color restoration
}

\author{
Matthieu Boffety and Frédéric Galland \\ Insitut Fresnel, CNRS, Aix-Marseille Université, École Centrale Marseille \\ Campus de Saint-Jérôme, 13013 Marseille, France
}

\begin{abstract}
Optical imaging plays an important role in oceanic science and engineering. However, the design of optical systems and image processing techniques for subsea environment are challenging tasks due to water turbidity. Marine snow is notably a major source of image degradation as it creates white bright spots that may strongly impact the performance of image processing methods. In this context, it is necessary to have a tool to foresee the behavior of these methods in marine conditions. This paper presents a phenomenological model of marine snow for image simulation. In order to highlight the interest of such a modeling for image processing characterization, the impact of marine snow perturbation on a color restoration technique is analyzed and a solution to improve the robustness of the algorithm is finally proposed.
\end{abstract}

\section{INTRODUCTION}

Optical imaging has become a valuable tool in the field of oceanic science and engineering [1]. However due to scattering and absorption properties of water imaging, system design and image processing in subsea environment remain challenging tasks and subjects of investigation and technological innovation [2], [3]. In this context, being able to foresee operating conditions and the behavior of image processing techniques in underwater environments is of great interest. Optical image simulation is therefore an attractive tool to tackle these issues [4].

Various approaches have already been proposed [5], [6] to simulate underwater images from a known scene. In order to obtain realistic underwater images it is notably important to take into account all phenomena involved in image degradation such as attenuation, scattering or noise phenomena. For the latter, besides classical Poisson and Gaussian noise models commonly considered in the field of photodetection (e.g for shot noise and thermal noise modeling), an other major source of degradation can be encountered in underwater images: The presence of white bright spots randomly distributed on the images. This phenomenon is generally designated as marine snow and is due to light reflection on small particle aggregates. Classical approaches of underwater image simulation assume an homogeneous effective medium and thus do not take into account the presence of local inhomogeneities that induces the phenomenon of marine snow. Nevertheless, this source of degradation has a great impact on visual image quality and its influence on image processing performances is yet to be addressed. Image simulation appears again to be a precious tool to deal with this issue.

This paper presents a phenomenological model of underwater image formation to take into account marine snow effect on color images. The purpose of this study is to illustrate the interest of such a model for image processing and to highlight the benefits one can obtain by using this kind of simulator for the development and characterization of image processing algorithms.

After this introduction, section II presents the model underlying our approach in the context of optical underwater imaging under artificial illumination. In the third part, in order to illustrate the interest of this simulator, the impact of marine snow on the behavior of a standard color restoration algorithm is shown allowing one to propose an improvement of the robustness of the method to the presence of marine snow.

\section{IMAGE SIMULATION IN THE PRESENCE OF MARINE SNOW}

\section{A. Brief recall of the Jaffe-McGlamery image formation model}

According to the classical Jaffe-McGlamery image formation model the irradiance measured by an underwater optical sensor can be written as [4], [5], [6]

$$
\begin{aligned}
\mathcal{E}_{\text {tot }, \lambda}(x, y, z)= & e^{-c_{\lambda} R_{c}} \mathcal{E}_{0, \lambda}(x, y, z) \\
& +\left[\mathcal{E}_{0, \lambda} * h_{\lambda}\right](x, y, z)+\mathcal{E}_{\mathrm{b}, \lambda}(x, y, z)
\end{aligned}
$$

where $*$ is the convolution operator over $(x, y), \mathcal{E}_{\text {tot, } \lambda}$ is the total measured irradiance on the CCD (in $\mathrm{W} \cdot \mathrm{m}^{-2} \cdot \mathrm{nm}^{-1}$ ), $\mathcal{E}_{0, \lambda}$ is the irradiance that would have been measured in the absence of water between the scene and the sensor, and $\mathcal{E}_{\mathrm{b}, \lambda}$ is the backscattered component [5] (i.e. light that has been scattered directly to the camera by the medium without reaching the scene). $R_{c}$ is the distance between the observed point $(x, y, z)$ and the camera (cf. Fig 1.(a)), $c_{\lambda}$ the beam extinction coefficient of the water and $h_{\lambda}$ its point spread function (PSF) as defined in [5], [6], [7].

Calculation of the backscattering component $\mathcal{E}_{\mathrm{b}, \lambda}$ is done by dividing the water column into thin slabs. Each of them are composed of small elementary volumes that scatter the light directly to the camera (cf. Fig 1.(b)). The contribution $\mathcal{E}_{\mathrm{k}, \lambda}$ of 

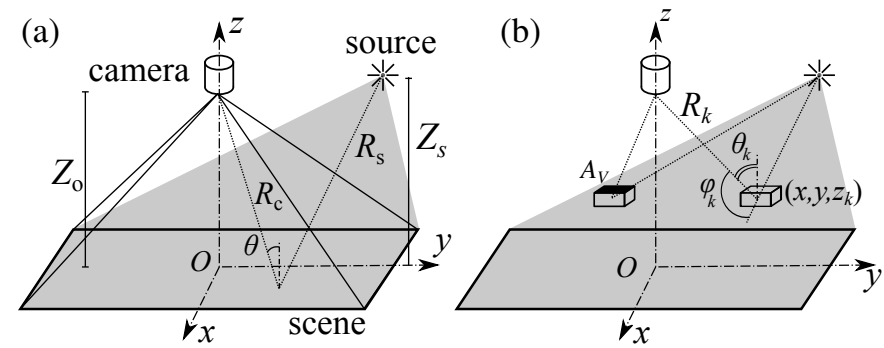

Fig. 1. Geometry and coordinate system of the model used for this study. The scene is assumed to be parallel to the detector plane. (a) For general calculation. (b) For backscattering component calculation.

each volume reads [5]

$$
\begin{aligned}
& \mathcal{E}_{\mathrm{k}, \lambda}\left(x, y, z_{k}\right)=\mathcal{E}_{\mathrm{k}, 0, \lambda}\left(x, y, z_{k}\right) e^{-c_{\lambda} R_{k}} \\
&+\left[\mathcal{E}_{\mathrm{k}, 0, \lambda} * h_{\lambda}\right]\left(x, y, z_{k}\right)
\end{aligned}
$$

where $R_{k}$ is the distance between the elementary volume and the CCD (cf. Fig. 1.(b)). $\mathcal{E}_{k, \lambda, 0}$ is the contribution of a volume without taking into account the medium between the volume and the camera and is calculated through the use of the water volume scattering function $\beta_{\lambda}$

$$
\begin{aligned}
\mathcal{E}_{k, \lambda, 0}\left(x, y, z_{k}\right)= & \frac{\mathcal{T}_{\lambda}}{4 N^{2}}\left(\frac{Z_{o}-F_{l}}{Z_{o}}\right)^{2} \\
& \times \beta_{\lambda}\left(\varphi_{k}\right) \mathcal{E}_{\mathrm{s}, \lambda}\left(x, y, z_{k}\right) \pi \cos ^{3} \theta_{k} \Delta z
\end{aligned}
$$

where $\mathcal{E}_{\mathrm{s}, \lambda}\left(x, y, z_{k}\right)$ is the illumination that reaches the volume, $\Delta z$ the height of the volume, $Z_{o}$ the object distance of the detector, and $F_{l}, N$, and $\mathcal{T}_{\lambda}$ are respectively the focal length, f-number and total transmission of the optical system lens.

Moreover these volumes are generally out of focus. The measured irradiance for a given depth $z_{k}$ is thus modified by the defocus point spread function $D_{\text {defocus. }}$ According to [8] $D_{\text {defocus }}$ can be modeled by a disk function of diameter $d$ given by

$$
d(z)=\frac{F_{l}^{2} z}{N\left(Z_{o}-z\right)\left(Z_{o}-F_{l}\right)}
$$

or by a Gaussian kernel of parameter $\sigma=\eta d$, where $\eta$ is a constant of proportionality depending on the characteristics of a given set-up [8]. In this study, a Gaussian kernel with parameter $\sigma=d / 2$ is considered. The final expression for the backscattering component is thus given by [5]

$$
\mathcal{E}_{\mathrm{b}, \lambda}(x, y)=\left.\sum_{k=1}^{z_{o} / \Delta z}\left[\mathcal{E}_{\mathrm{k}, \lambda} * D_{\text {defocus }}\right]\left(x, y, z_{k}\right)\right|_{z_{k}=\Delta z \times(k-1 / 2)}
$$

The discretization step $\Delta z$ has to be chosen small enough to verify the convergence of the sum of equation (4).

\section{B. A phenomenological approach of marine snow noise}

The modeling of marine snow properties is still a subject under investigation [9]. Nevertheless, for image simulation purposes, a simple way to model marine snow effect would be to generate a salt and pepper noise on the final image $\mathcal{E}_{\text {tot }, \lambda}$. However this model does not take into account various physical parameters such as the effect of water absorption and scattering on the signal backscattered by the particles, the size and shape of the particles or the defocus effect.

In the proposed approach and for simplification purpose, these macro-particles are assumed to behave like white lambertian scatterers. The spatial profile of their reflection coefficient is chosen to be a Gaussian function with standard deviation parameter $\sigma_{s}$ and maximum value equal to $R_{m}^{\lambda}$. This choice over the shape seems realistic to take into account that marine snow particles may be thicker in their center than in their circumference, however other choices could have been made and implemented in the simulator (such as a disk shape of radius $\sigma_{s}$ or other more complex shapes). Therefore the reflectance map of a given macro-particle located at pixel $\left(x_{0}, y_{0}\right)$ is given by

$$
\mathcal{R}_{\left[x_{0}, y_{0}\right]}^{\lambda}(x, y)=\alpha R_{m}^{\lambda} \exp \left(-\frac{\left[x-x_{0}\right]^{2}+\left[y-y_{0}\right]^{2}}{2 \sigma_{s}^{2}}\right)
$$

if $\sqrt{\left[x-x_{0}\right]^{2}+\left[y-y_{0}\right]^{2}} \leq 3 \sigma_{s}$ and 0 elsewhere. The coefficient $\alpha$ is defined so that

$$
\begin{aligned}
& \sum_{x, y} \mathcal{R}_{\left[x_{0}, y_{0}\right]}^{\lambda}(x, y) A_{V}= \\
& \int_{-\infty}^{\infty} \int_{-\infty}^{\infty} R_{m}^{\lambda} \exp \left(-\frac{\left[x-x_{0}\right]^{2}+\left[y-y_{0}\right]^{2}}{2 \sigma_{s}^{2}}\right) \mathrm{d} x \mathrm{~d} y
\end{aligned}
$$

where $A_{V}$ is the surface associated with an elementary volume considered in the backscattering calculation (cf. Fig 1.(b)). This definition of $\alpha$ is chosen to avoid discretization problem when the particle size becomes smaller than a pixel. It leads to

$$
\alpha=2 \pi \frac{\sigma_{s}^{2}}{A_{V}}\left[\sum_{x, y} \exp \left(-\frac{\left[x-x_{0}\right]^{2}+\left[y-y_{0}\right]^{2}}{2 \sigma_{s}^{2}}\right)\right]^{-1}
$$

Then, for each slab used to compute the backscattering component $\mathcal{E}_{\mathrm{b}, \lambda}$, a global reflectance map is created. This map is generated by positioning the white Gaussian-shaped particles according to a salt and pepper distribution: For each slab elementary volume $V=A_{V} \times \Delta z$, there is a probability $p=C_{p} \times V$ of finding a particle in the volume, where $C_{p}$ is the concentration in particles. This model assumes that only one macro-particle can be found in a given discretization volume of the slab. Therefore the discretization of the water column has also to be chosen accordingly ${ }^{1}$.

Let $\mathcal{R}_{\lambda, k}^{\text {snow }}$ be the created reflectance map due to marine associated with the $k^{t h}$ slab. The resulting irradiance $\mathcal{E}_{k, 0, \lambda}^{\text {snow }}$, that would have been observed on the sensor without taking

\footnotetext{
${ }^{1}$ In the case where 2 particles located on neighboring pixels $\left(x_{0}, y_{0}\right)$ and $\left(x_{1}, y_{1}\right)$ overlap, it will be assumed that the corresponding reflectance map is simply equal to the maximum of the 2 reflectivities $\max \left\{\mathcal{R}_{\left[x_{0}, y_{0}\right]}^{\lambda}(x, y), \mathcal{R}_{\left[x_{1}, y_{1}\right]}^{\lambda}(x, y)\right\}$, i.e. the brightest particle hides the other one.
} 
into account the medium between the slab and the camera, is then computed according to the equation:

$$
\begin{aligned}
\mathcal{E}_{k, 0, \lambda}^{\text {snow }}\left(x, y, z_{k}\right)= & \frac{\mathcal{T}_{\lambda}}{4 N^{2}}\left(\frac{Z_{o}-F_{l}}{Z_{o}}\right)^{2} \\
& \times \mathcal{R}_{\lambda, k}^{\text {snow }}(x, y) \mathcal{E}_{s, \lambda}\left(x, y, z_{k}\right) \cos ^{4} \theta_{k}
\end{aligned}
$$

The signal is then modified according to the water PSF:

$$
\begin{aligned}
\mathcal{E}_{k, \lambda}^{\text {snow }}\left(x, y, z_{k}\right)=\mathcal{E}_{k, 0, \lambda}^{\text {snow }}\left(x, y, z_{k}\right) e^{-c_{\lambda} R_{k}} & \\
& +\left[\mathcal{E}_{k, 0, \lambda}^{\text {snow }} * h_{\lambda}\right]\left(x, y, z_{k}\right)
\end{aligned}
$$

The final expression of the backscattering component is thus

$$
\begin{aligned}
\mathcal{E}_{\mathrm{b}, \lambda}(x, y)=\sum_{k=1}^{Z_{o} / \Delta z}\left[\left(\mathcal{E}_{\mathrm{k}, \lambda}+\mathcal{E}_{k, \lambda}^{\text {snow }}\right)\right. \\
\left.* D_{\text {defocus }}\right]\left.\left(x, y, z_{k}\right)\right|_{z_{k}=\Delta z \times(k-1 / 2)}
\end{aligned}
$$

The signal backscattered by the particles is therefore affected by the medium as well as by defocus defect.

\section{Experimental set-up and examples of images}

In this study, two types of water are considered: a clear water type and a turbid one. Their absorption, scattering and extinction coefficients, respectively $a_{\lambda}, b_{\lambda}$ and $c_{\lambda}$ (with $c_{\lambda}=a_{\lambda}+b_{\lambda}$ ), are given by Fig. 2 over a 400-800nm spectral interval. The detection system was assumed to be a CCD camera equipped with a lens of focal length $F_{l}=27.5 \mathrm{~mm}$, f-number $N=2.8$ and transmission $\mathcal{T}_{\lambda}=1$ over the whole spectrum. The medium is illuminated by an isotropic punctual white source with unitary power and translated from the camera by $1 \mathrm{~m}$ in the $y$ direction (cf.Fig. 1). The discretization of the water column is chosen to have $\Delta z=5 \mathrm{~cm}$. Moreover it is assumed that the RGB images are acquired through a camera with spectral responses given by Fig. 4.

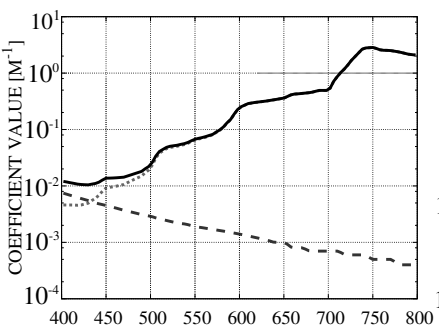

(a) WAVELENGTH [NM]

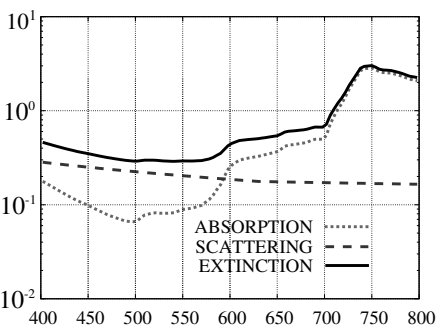

(b) WAVELENGTH [NM]
Fig. 2. Inherent optical properties spectra of the types of water used in this article. The absorption beam coefficient is plotted in light gray dot line, the scattering in dark gray dashed line, and the total extinction coefficient in black plain line. (a) For clear water (referred in the text as "type 1"). (b) For turbid water (referred in the text as "type 2 ").

Fig. 3 shows examples of simulated backscattering component with marine snow for both types of water. Images were generated using the marine snow phenomenological model previously described, assuming the scatterers have a Gaussian shape of size parameter equal to $\sigma_{s}=0.5 \mathrm{~mm}$, a reflexion coefficient of $R_{m}^{\lambda}=1$ over the whole spectrum, and assuming that the particle concentration is equal to $10 \mathrm{~m}^{-3}$ (Fig. 3.(c) and (d)), $25 \mathrm{~m}^{-3}$ (Fig. 3.(e) and (f)) and $50 \mathrm{~m}^{-3}$ (Fig. 3.(g) and (h)).

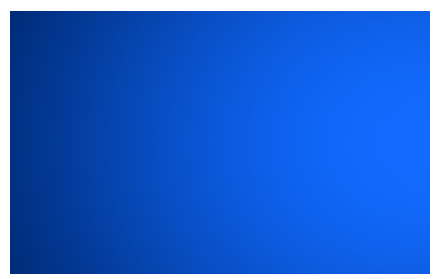

(a)

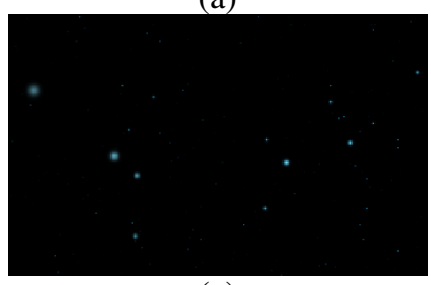

(c)

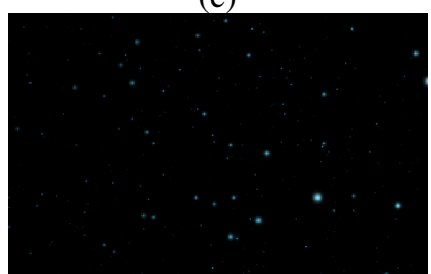

(e)

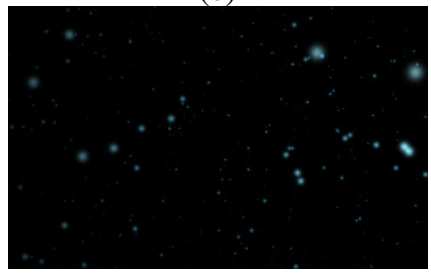

(g)

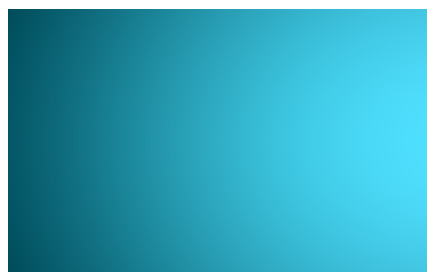

(b)

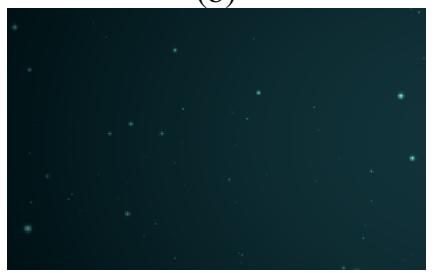

(d)

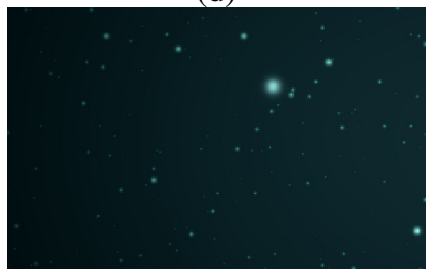

(f)

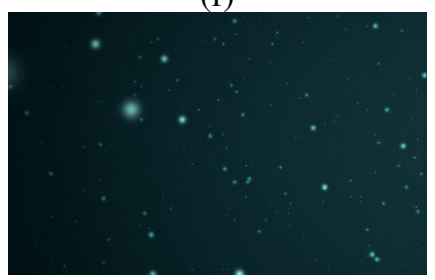

(h)
Fig. 3. Effect of marine snow on the backscattering component (i.e. the image that would have been observed if the scene were located sufficiently far from the sensor). Left column: Type 1 water. Right column: Type 2 water. (a)-(b) Image without marine snow. (c)-(d) Image of marine snow for a particle concentration equal to $10 \mathrm{~m}^{-3}$. (e)-(f) Idem for particle concentration = $25 \mathrm{~m}^{-3}$. (g)-(h) Idem for particle concentration $=50 \mathrm{~m}^{-3}$. Image size is $256 \times 160$.

As we can see, in both cases the marine snow appears blueish as it is affected by the water absorption and diffusion. The size difference of the white "hot spots" is mainly due to defocus when particle are close to the sensor. Moreover for type 1 water the signal due to marine snow is stronger than the backscattering signal of the medium. It is thus expected to be in this case the main source of degradation of the image whereas the backscattering of the medium is still visible for water of type 2, which means that both effects will have to be taken into consideration.

\section{A VALUABLE TOOL FOR IMAGE PROCESSING}

The primary goal of the Jaffe-McGlamery image simulation tool for underwater optics was to design optimal underwater systems [5], [6], but simplified versions were also used for 


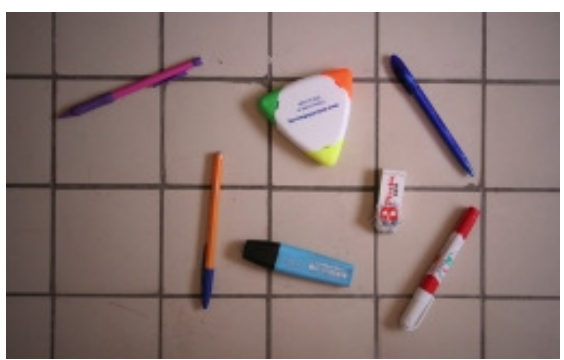

(a)

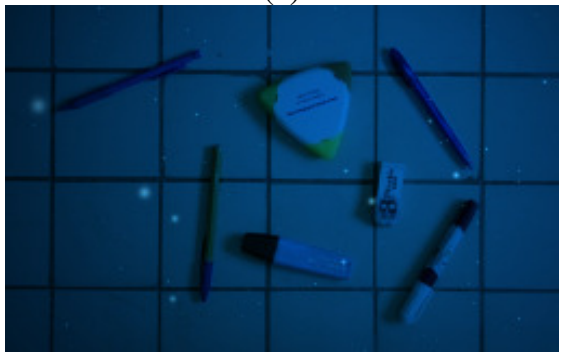

(d)

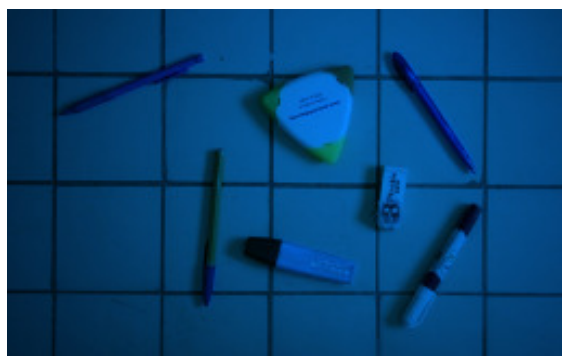

(b)

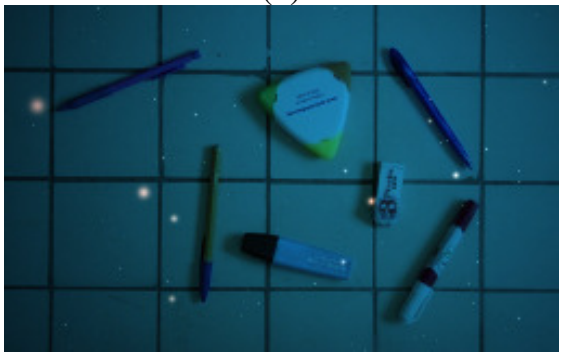

(e)

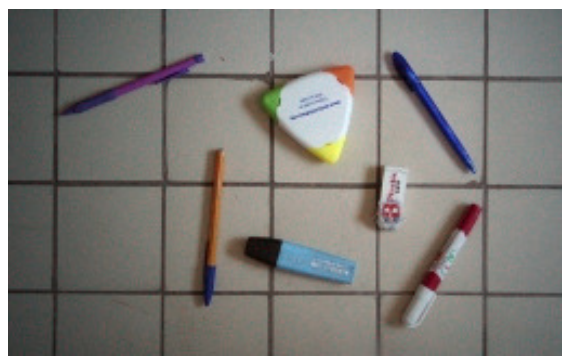

(c)

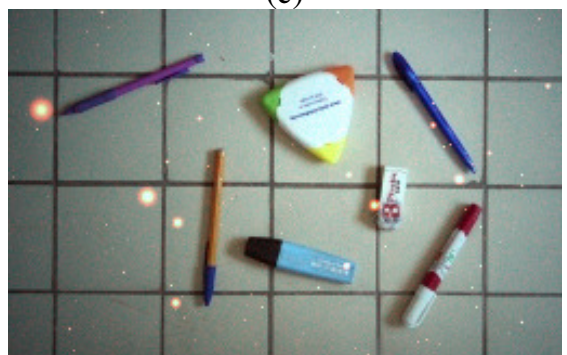

(f)

Fig. 5. Restoration of an underwater image generated with the simulator. The water is assumed to have properties of type 1 and the scene to be at $7 \mathrm{~m}$ from the camera. Image size is $256 \times 160$. (a) Simulated image assuming the scene is in the air. (b) Idem but in underwater conditions. (c) Restoration of the underwater image (b) using the HSM algorithm. (d) Simulated underwater image as in (b) but in the presence of marine snow. (e) Restoration of image (d) using the HSM algorithm. (f) Idem but using the robust HSM version.

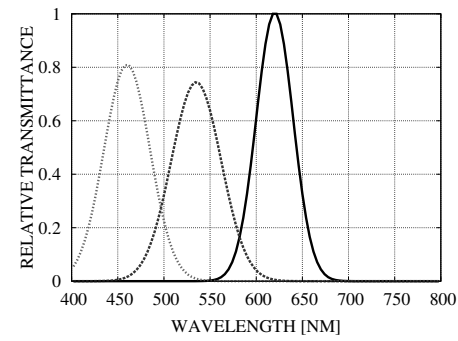

Fig. 4. RGB spectral responses of the camera used to generate the images in this study.

characterization and improvement of image restoration algorithms [10], [11]. In this section, we show the interest of underwater image simulation to study color image restoration algorithms. Indeed, color degradation and restoration are major aspects of underwater imaging and are the subject of various studies [4], [12], [13], [14], [15].

\section{A. An example of image color restoration algorithm}

Assuming that RGB images are acquired with the same camera as in section II (Fig. 4), two synthetic images of a given scene have been simulated. Fig. 5.(a) presents the image that would have been acquired in the air, whereas Fig. 5.(a) corresponds to the image that would have been observed in underwater conditions. It assumes type 1 water properties and a scene-camera distance of $7 \mathrm{~m}$. In order to take into account sensor noises, the 3 RGB channels of the image have been corrupted with an additive Gaussian noise with the same standard deviation $\sigma$ set to $0.1 \%$ of the maximum value of the 3 channels. It is clear on Fig. 5.(b) that the spectral information of the scene has been modified by the medium.

To restore the initial colors of the image, it is proposed here to use and study the behavior of a simplified version of an histogram stretching method [12] (HSM), which is a technique belonging to the canonical gain/offset type [16]. Indeed although this technique is quite simple, it generally leads to interesting color restorations. Let $\mathbf{I}(x, y)=$ $\left[I_{R}(x, y), I_{G}(x, y), I_{B}(x, y)\right]^{T}$ be the initial measured RGB image and $\mathbf{J}(x, y)=\left[J_{R}(x, y), J_{G}(x, y), J_{B}(x, y)\right]^{T}$ be the RGB restored image, where $J_{c}$ and $I_{c}$ are the image components in channel $c \in\{R, G, B\}$, and ${ }^{T}$ is the transpose operator. According to the HSM restoration method, $J_{c}$ is estimated as

$$
J_{c}(x, y)=\frac{255}{\max _{x, y}\left(I_{c}\right)-\min _{x, y}\left(I_{c}\right)}\left[I_{c}(x, y)-\min _{x, y}\left(I_{c}\right)\right]
$$

It can be seen on Fig. 5.(c) that even at 7m, where colors are severely degraded, HSM leads to very interesting color restoration.

\section{B. Impact of marine snow on restorations}

On the contrary, when applied to the real underwater image of Fig. 6.(a), the method gives very disappointing results: The restored image (Fig. 6.(b)) remains dark and blueish. An hypothesis would be that the bright spots of marine snow in the image limit the dynamic of the reconstruction. Using the phenomenological model introduced in the previous section, it is possible to add such a perturbation on the simulated image.

Fig. 5.(d) presents the result of the simulation when marine snow has been added to Fig. 5.(b) assuming the scatterers have a Gaussian shape of size parameter equal to $\sigma_{s}=0.5 \mathrm{~mm}$, a reflexion coefficient of $R_{m}^{\lambda}=1$ over the whole spectrum 


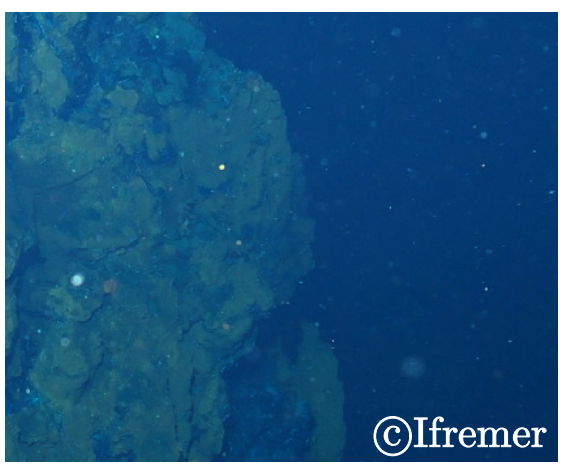

(a)

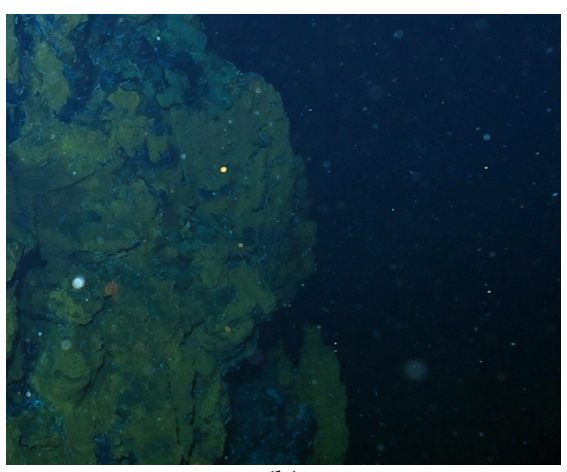

(b)

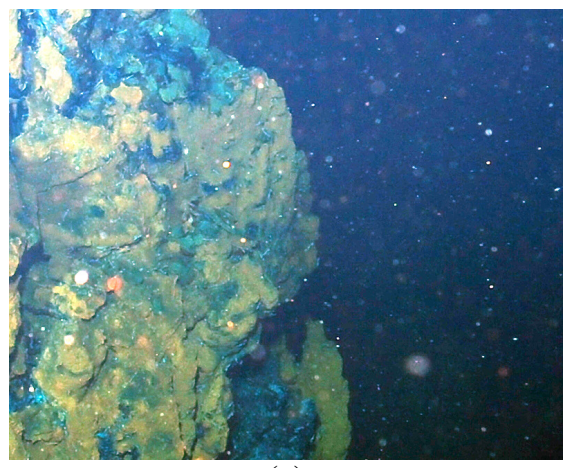

(c)

Fig. 6. Examples of color restoration on real data. (a) Underwater image $(801 \times 651$ pixel extract) provided by Ifremer. (b) Restoration with HSM. (c) Restoration with robust HSM.

and that the particle concentration is equal to $10 \mathrm{~m}^{-3}$. When applying the HSM color restoration algorithm on this image, the same behavior as on Fig. 6.(b) can be observed (cf. Fig. 5.(e)), i.e. the image remains dark and blueish. Therefore marine snow seems to be indeed what is limiting the HSM algorithm in Fig. 6.(b).

\section{Improvement of the algorithm}

Since HSM relies on calculations of the minimum and maximum values of the 3 RGB channels, the robustness of the algorithm to the presence of marine snow can be improved by replacing calculation of the extrema by calculation of the quantiles. More precisely in our case, the maximum value is replaced by the $99 \%$-quantile value (i.e. the intensity value such that $1 \%$ of the highest values are not taken into account). This choice relies on the fact that the high intensity values of the image seem to correspond to the marine snow bright spots. When applying this robust version of the HSM on the simulated image we get the result of Fig 5.(f), which is close to the result obtained in the absence of marine snow. When applied to the real image of Fig. 6.(a), this robust HSM allows one to recover the yellow hue of the rock (cf. Fig. 6.(c)).

\section{CONCLUSION}

It has been shown that marine snow is an important perturbation that can strongly decrease the performance of color restoration algorithms. It is therefore necessary to be able to characterize the behavior of such algorithms in the presence of such a phenomenon. In this context, a phenomenological model allowing the simulation of underwater images in presence of marine snow has been presented. The behavior of a standard HSM color restoration technique has then been analyzed in the presence of marine snow, allowing one to increase its robustness to the presence of this perturbation in the perspectives of real data application.

The immediate perspective of this work is to generalize this approach in order to automatically determine which is the best quantile value to use in the robust version of the color restoration algorithm. Moreover the interest of such a simulator is not limited to color restoration and can be used to characterize the robustness of other image processing algorithms to the presence of marine snow.

\section{ACKNOWLEDGMENT}

The authors would like to thank Philippe Réfrégier from Institut Fresnel and Anne-Gaëlle Allais from Ifremer for fruitful collaborations and discussions, and Ifremer for providing the underwater image. This work has been partially supported by French National Research Agency (ANR) through COSINUS program (project OSIFIOST $\mathrm{n}^{\circ}$ ANR-08-COSI-008), with an additional support through the Pôle Mer PACA.

\section{REFERENCES}

[1] J. Jaffe, J. Mclean, M. Strand, and K. Moore, Underwater Optical Imaging: Status and Prospects, Oceanography 14, 64-75 (2001).

[2] D. Kocak, F. Dalgleish, and F. Caimi, A focus on recent developments and trends in underwater imaging, Marine Technology 42, 52-67 (2008).

[3] W. Hou, A. Weidemann, and D. Gray, Improving Underwater Imaging with Ocean Optics Research, Ocean Science and Technology pp. 195-196 (2008).

[4] R. Schettini and S. Corchs, Underwater Image Processing: State of the Art of Restoration and Image Enhancement Methods, EURASIP Journal on Advances in Signal Processing 2010 (2010).

[5] B. McGlamery, Computer analysis and simulation of underwater camera system performance, Tech. rep., Scripps. Inst. of Oceanography, San Diego (1975).

[6] J. Jaffe, Computer Modeling and the Design of Optimal Underwater Imaging Systems, IEEE Journal of Oceanic Engineering 15, 101-111 (1990).

[7] S. Negahdaripour, H. Zhang, and X. Han, Investigation of photometric stereo method for 3-d shape recovery from underwater imagery, in MTS/IEEE Oceans, (Marine Technol. Soc, Biloxi, 2002), pp. 1010-1017.

[8] A. Pentland, A new sense for depth of field, IEEE transactions on pattern analysis and machine intelligence 9, 523-531 (1987).

[9] W. Slade, E. Boss, and C. Russo, Effects of particle aggregation and disaggregation on their inherent optical properties, Optics Express 19, $7945-7959$ (2011).

[10] Y. Schechner and N. Karpel, Recovery of Underwater Visibility and Structure by Polarization Analysis, IEEE Journal of Oceanic Engineering 30, 570-587 (2005).

[11] E. Trucco and A. Olmos-Antillon, Self-Tuning Underwater Image Restoration, IEEE Journal of Oceanic Engineering 31, 511-519 (2006).

[12] K. Iqbal, R. Abdul Salam, M. Osman, and A. Talib, Underwater Image Enhancement Using An Integrated Colour Model. IAENG International Journal of Computer Science 34, 239-244 (2007).

[13] J. Åhlén, E. Bengtsson, and D. Sundgren, Evaluation of underwater spectral data for colour correction applications, in Proceedings of the 5th WSEAS International Conference on Circuits, Systems, Electronics, Control \& Signal Processing, (World Scientific and Engineering Academy and Society (WSEAS), Dallas, 2006), pp. 321-326. 
[14] L. Torres-Méndez and G. Dudek, A statistical learning-based method for color correction of underwater images, Research on Computer Science 17, 10 (2005).

[15] M. Chambah, D. Semani, A. Renouf, P. Courtellemont, and A. Rizzi, Underwater color constancy: enhancement of automatic live fish recognition, Color Imaging IX: Processing, Hardcopy, and Applications 5293, 157-168 (2003).

[16] Z.-U. Rahman, G. Woodell, and D. Jobson, A Comparison of the Multiscale Retinex With Other Image Enhancement Techniques, in IS\&Ts 50th Annual Conference: A Celebration of All Imaging, (Citeseer, 1997), pp. $426-432$. 